\title{
Isometric approximation property of unbounded sets
}

\author{
Jussi Väisälä
}

\begin{abstract}
We give a necessary and sufficient quantitative geometric condition for an unbounded set $A \subset \mathrm{R}^{n}$ to have the following property with a given $c>0$ : For every $\varepsilon \geq 0$ and for every map $f: A \rightarrow \mathrm{R}^{n}$ such that ||$f x-f y|-| x-y|| \leq \varepsilon$ for all $x, y \in A$, there is an isometry $T: A \rightarrow \mathrm{R}^{n}$ such that $|T x-f x| \leq c \varepsilon$ for all $x \in A$.
\end{abstract}

2000 Mathematics Subject Classification: 46C05, 46B20, 30C65

\section{Introduction}

1.1. Nearisometries. Let $E$ and $F$ be real Hilbert spaces and let $A \subset E$. A map $f: A \rightarrow F$ is said to be a nearisometry if there is $\varepsilon \geq 0$ such that

$$
|x-y|-\varepsilon \leq|f x-f y| \leq|x-y|+\varepsilon
$$

for all $x, y \in A$. More precisely, such a map is an $\varepsilon$-nearisometry. In the literature, the $\varepsilon$-nearisometries are often called $\varepsilon$-isometries.

We study the stability question: How well can an $\varepsilon$-nearisometry be approximated by a true isometry? The following fundamental result was proved by D.H. Hyers and S.M. Ulam HU] in 1945: If $f: E \rightarrow F$ is a surjective $\varepsilon$-nearisometry, then there is a surjective isometry $T: E \rightarrow F$ with

$$
d(T, f)=\sup \{|T x-f x|: x \in E\} \leq 10 \varepsilon .
$$


The bound $10 \varepsilon$ has later been improved to $\sqrt{2} \varepsilon$; see $[\overline{S e}, 1.2]$. For a Banach space version, see $[\mathrm{BL}, 15.2]$. In the case $E=F=\mathrm{R}^{n}$ the result holds without the surjectivity condition, as proved by R. Bhatia and P. Šmrl [BS, Th.1]. A survey of nearisometries is given in $\mathrm{Vä2}$.

The case where $A \subset E=F=\mathrm{R}^{n}$ has been studied in [ATV] and in [Vä1]. For $c>0$ we say that a set $A \subset \mathrm{R}^{n}$ has the $c$-isometric approximation property, abbreviated $c$-IAP, if for each $\varepsilon \geq 0$ and for each $\varepsilon$-nearisometry $f: A \rightarrow \mathrm{R}^{n}$ there is an isometry $T: \mathrm{R}^{n} \rightarrow \mathrm{R}^{n}$ such that

$$
d(T, f)=\sup \{|T x-f x|: x \in A\} \leq c \varepsilon .
$$

If $A$ has the $c$-IAP and contains at least two points, then clearly $c \geq 1 / 2$.

The whole space $\mathrm{R}^{n}$ has the $\sqrt{2}$-IAP. In $[\mathrm{Vä1}, 2.5]$ we gave a quantitative geometric characterization for bounded subsets of $\mathrm{R}^{n}$ with the $c$-IAP in terms of the so-called $c$-solar systems. For sets without isolated points, it can be expressed in the following simple form. For a unit vector $u \in \mathrm{R}^{n}$ let $\pi_{u}: \mathrm{R}^{n} \rightarrow \mathrm{R}$ be the projection $\pi_{u} x=x \cdot u$. The thickness of a bounded set $A \subset \mathrm{R}^{n}$ is the number

$$
\theta(A)=\inf \left\{d\left(\pi_{u} A\right):|u|=1\right\}
$$

where $d$ denotes diameter. Then $A$ has the $c$-IAP if and only if $\theta(A) \geq d(A) / c^{\prime}$, where $c$ and $c^{\prime}$ depend only on each other and on $n$. In particular, a ball has the $c$-IAP with $c=c(n)$; this was essentially proved by F. John Jo already in 1961 . From a recent result of E. Matoušková [Ma, 4.1] it follows that $c(n)$ cannot be chosen to be independent of $n$.

The motivation for the present paper was to find a characterization for the unbounded subsets of $\mathrm{R}^{n}$ with the $c$-IAP. It turns out that the answer is simpler than in the case of bounded sets, and it is given in 2.3. We associate to each unbounded set $A \subset \mathrm{R}^{n}$ a number $\mu(A) \in[0,1]$ and show that if $A$ has the $c$-IAP, then $\mu(A) \geq 1 / c^{\prime} \geq 0$ with $c^{\prime}=17 c$. Conversely, if $\mu(A) \geq 1 / c^{\prime}$, then $A$ has the $c$-IAP with $c=\sqrt{2} c^{\prime}$. The constant $\sqrt{2}$ is the best possible.

Contrary to the bounded case, these estimates do not depend on the dimension $n$. In fact, several arguments are valid in arbitrary Hilbert spaces, but the most satisfactory formulation is obtained in the euclidean $n$-space $\mathrm{R}^{n}$.

1.2. Summary of the paper. We start by introducing in Section 2 the number $\mu(A)$ and other auxiliary concepts needed in the proof. We can then state the main theorem 2.3 and various related remarks. In Section 3 we show that $c$ IAP implies the property $\mu(A) \geq 1 / c^{\prime}$. The converse is proved in Section 4 , where we give a more general result valid for all Hilbert spaces, which may have independent interest. In Section 5 we give a short elementary proof and an improvement of the original Hyers-Ulam theorem. A remark on the Banach space case is given in Section 6.

\section{Formulation of the main result}


2.1. Notation. Let $E$ be a real Hilbert space. We let $x \cdot y$ denote the inner product of vectors $x, y \in E$, and the norm of $x$ is $|x|=\sqrt{x \cdot x}$. Open and closed balls with center $x$ and radius $r$ are written as $B(x, r)$ and $\bar{B}(x, r)$, respectively, and we abbreviate $B(r)=B(0, r), \bar{B}(r)=\bar{B}(0, r)$. The unit sphere is $S(1)=\partial B(1)$. The diameter of a set $A \subset E$ is $d(A)$, and the distance between nonempty sets $A, B \subset E$ is $d(A, B)$. The standard basis of $\mathrm{R}^{n}$ is $e_{1}, \ldots, e_{n}$. The central projection $p: E \backslash\{0\} \rightarrow S(1)$ is defined by

$$
p x=x /|x| .
$$

An expression like $a b / c d e$ means $(a b) /(c d e)$. To simplify expressions we often omit parentheses writing $f x=f(x)$ etc.

2.2. Definitions. Let $A \subset E$ be an unbounded set and let $u \in E$ be a unit vector. We say that a sequence $\left(x_{j}\right)$ in $A$ converges directionally to $u$ if $\left|x_{j}\right| \rightarrow \infty$ and $p x_{j} \rightarrow u$. A unit vector $u$ is a cluster direction of $A$ if there is a sequence in $A$ converging directionally to $u$. We let $\operatorname{cd} A$ denote the set of all cluster directions of $A$. Equivalently,

$$
\operatorname{cd} A=\bigcap\{\operatorname{cl} p[A \backslash B(R)]: R>0\} .
$$

Obviously $\operatorname{cd}(A+b)=\operatorname{cd} A$ for each $b \in E$. If $\operatorname{dim} E<\infty$, the $\operatorname{set} \operatorname{cd} A$ is compact and nonempty.

Let $X \subset S(1)$. For $e \in S(1)$ we set

$$
\sigma(e, X)=\sup \{|u \cdot e|: u \in X\}, \quad \mu_{1}(X)=\inf \{\sigma(e, X):|e|=1\}
$$

with the convention $\mu_{1}(\emptyset)=0$. Thus $\mu_{1}(X)$ is small if and only if $X$ lies in a narrow neighborhood of a hyperplane through the origin. In fact, $\mu_{1}(X)=$ $\theta(X \cup(-X)) / 2$.

For an unbounded set $A \subset E$ we set

$$
\mu(A)=\mu_{1}(\operatorname{cd} A) .
$$

For alternative definitions of $\mu(A)$ for $A \subset \mathrm{R}^{n}$, see 2.7 .

We can now state the main result of the paper:

2.3. Main theorem. For an unbounded set $A \subset \mathrm{R}^{n}$, the following conditions are quantitatively equivalent:

(1) A has the $c$-IAP.

(2) $\mu(A) \geq 1 / c^{\prime}$.

More precisely, (1) implies (2) with $c^{\prime}=17 c$, and (2) implies (1) with $c=\sqrt{2} c^{\prime}$. The constant $\sqrt{2}$ is the best possible.

Part $(1) \Rightarrow(2)$ will be proved in Section 3, and the converse part in Section 4. Observe that the bounds in 2.3 do not depend on $n$. 
2.4. Remarks. 1 . Always $0 \leq \mu(A) \leq 1$.

2. The number $\mu(A)$ is invariant under translations: $\mu(A+b)=\mu(A)$ for each $b \in \mathrm{R}^{n}$.

3. If $A \subset A^{\prime}$, then $\mu(A) \leq \mu\left(A^{\prime}\right)$.

4. If $A$ contains a half space, then $\mu(A)=1$.

5. For $e \in S(1)$ and $0 \leq \alpha \leq \pi / 2$, let $C(e, \alpha)$ be the cone $\{x \in E: x \cdot e \geq$ $|x| \cos \alpha\}$. Then $\mu(C(e, \alpha))=\sin \alpha$.

6. Since $\mu\left(\mathrm{R}^{n}\right)=1$, the Bhatia-Šemrl result mentioned in 1.1 follows from 2.3 as a corollary.

2.5. Sharpness. The bound $c=\sqrt{2} c^{\prime}$ in 2.3 is the best possible. In fact, for each $n$ there is a 1-nearisometry $f: \mathrm{R}^{n} \rightarrow \mathrm{R}^{n}$ such that $d(T, f)$ is at least the Jung constant $J\left(\mathrm{R}^{n}\right)=\sqrt{2 n /(n+1)}$ of $\mathrm{R}^{n}$ for every isometry $T: \mathrm{R}^{n} \rightarrow \mathrm{R}^{n}$; see [HV], 1.11]. Hence the whole space $\mathrm{R}^{n}$ does not have the $c$-IAP for any $c<J\left(\mathrm{R}^{n}\right)$. Since $\mu\left(\mathrm{R}^{n}\right)=1$, the number $\sqrt{2}$ cannot be replaced by any smaller universal constant.

On the other hand, the bound $17 c$ is presumably far from optimal. We get a lower estimate for this bound by considering the set $A=\mathrm{R}$, which has the 1-IAP in $\mathrm{R}$. Since $\mu(A)=1$, Theorem 2.3 is not true if the number 17 is replaced by any universal constant less than 1 .

2.6. Uniqueness. Suppose that $A \subset \mathrm{R}^{n}$ is an unbounded set with the $c$-IAP. If $f: A \rightarrow \mathrm{R}^{n}$ is an $\varepsilon$-nearisometry, then the isometry $T$ with $d(T, f) \leq c \varepsilon$ given by the $c$-IAP is uniquely determined up to translation. To prove this, it suffices to show that there is at most one linear isometry $U: \mathrm{R}^{n} \rightarrow \mathrm{R}^{n}$ with $d(U, f) \leq K<\infty$. Assume that $U$ and $U^{\prime}$ are such maps. Let $e \in \operatorname{cd} A$. Since $\mu(A)>0$, the set $\operatorname{cd} A$ spans $\mathrm{R}^{n}$. Hence it suffices to show that $U e=U^{\prime} e$.

Choose a sequence $\left(x_{j}\right)$ in $A$ converging directionally to $e$. Since $\left|U x-U^{\prime} x\right| \leq$ $2 K$ for all $x \in A$, we get $\left|U\left(p x_{j}\right)-U^{\prime}\left(p x_{j}\right)\right| \leq 2 K /\left|x_{j}\right|$. Letting $j \rightarrow \infty$ yields $U e-U^{\prime} e=0$ as desired.

2.7. Alternative definitions for $\mu(A)$. The following considerations may clarify the meaning of $\mu(A)$, but they are not actually needed in the paper.

Let $E$ be a Hilbert space, let $e \in E$ be a unit vector, and let $0 \leq \alpha \leq \pi / 2$. We let $D(e, \alpha)$ denote the open double cone with axis span $e$, vertex 0 and central angle $\alpha$ :

$$
D(e, \alpha)=\left\{x \in \mathrm{R}^{n}:|x \cdot e|>|x| \cos \alpha\right\}=\bigcup\{B(t e,|t| \sin \alpha): t \in \mathrm{R}\} .
$$

For an unbounded set $A \subset E$ we write

$$
\begin{aligned}
& \varphi(A)=\sup \{\alpha: A \cap D(e, \alpha) \text { is bounded for some } e \in S(1)\} \\
& \tau(A)=\sup _{|e|=1} \liminf _{|t| \rightarrow \infty} d(t e, A) /|t| .
\end{aligned}
$$


2.8. Proposition. Always $\tau(A)=\sin \varphi(A), \mu(A) \leq \cos \varphi(A)$. If $\operatorname{dim} E<\infty$, then $\mu(A)=\cos \varphi(A)=\sqrt{1-\tau(A)^{2}}$.

We omit the easy proof. The inequality $\mu(A) \geq \cos \varphi(A)$ for $E=\mathrm{R}^{n}$ follows from 3.1 below.

\section{Proof for $(1) \Rightarrow(2)$}

To obtain the part $(1) \Rightarrow(2)$ of 2.3 we define an auxiliary map and prove several lemmas.

3.1. Lemma. Suppose that $L \subset \mathrm{R}^{n}$ is a linear subspace, and let $P: \mathrm{R}^{n} \rightarrow L$ and $P^{\prime}: \mathrm{R}^{n} \rightarrow L^{\perp}$ be the orthogonal projections. Suppose also that $A \subset \mathrm{R}^{n}$ is an unbounded set such that that $\left|P^{\prime} u\right|<q \leq 1$ for all $u \in \operatorname{cd} A$. Then the set $A \cap\left\{x:\left|P^{\prime} x\right| \geq q|x|\right\}$ is bounded.

Proof. If the lemma is false, there is a sequence $\left(x_{j}\right)$ in $A$ converging directionally to $u \in \operatorname{cd} A$ such that $\left|P^{\prime} x_{j}\right| \geq q\left|x_{j}\right|$ for all $j$. Dividing by $\left|x_{j}\right|$ and letting $j \rightarrow \infty$ yields $\left|P^{\prime} u\right| \geq q$, a contradiction.

3.2. Remarks. 1. The bounded set in 3.1 can also be written as $A \cap\left\{x:\left|P^{\prime} x\right| \geq\right.$ $r|P x|\}$, where $r=q / \sqrt{1-q^{2}}$.

2. Lemma 3.1 does not hold in infinite-dimensional Hilbert spaces, because an unbounded set need not have any cluster directions. This is the main reason why we consider in this section only the case $E=\mathrm{R}^{n}$.

3.3. An auxiliary map. In 3.3 3.11 we assume that $n \geq 2$ and write $\mathrm{R}^{n}=$ $\mathrm{R}^{n-1} \times \mathrm{R}$. Given a number $M \geq 1$ we define a map $g: \mathrm{R}^{n} \rightarrow \mathrm{R}^{n}$ by

$$
g(x, t)= \begin{cases}(x, t+\sqrt{|x|}) & \text { for }|x| \leq M, \\ (x, t+\sqrt{M}) & \text { for }|x| \geq M .\end{cases}
$$

We want to show that $g$ is a 1-nearisometry in a certain set. For that purpose, let $z=(x, t), z^{\prime}=\left(x^{\prime}, t^{\prime}\right) \in \mathrm{R}^{n}$. Set

$$
d=\left|z^{\prime}-z\right|, \quad D=\left|g z^{\prime}-g z\right|, h=\left|x^{\prime}-x\right| .
$$

3.4. Lemma. If $|x| \leq\left|x^{\prime}\right| \leq M, r=1 / 8 \sqrt{M},|t| \leq r\left|x^{\prime}\right|$ and $\left|t^{\prime}\right| \leq 3 r\left|x^{\prime}\right|$, then $|D-d| \leq 1$.

Proof. A direct computation gives $D^{2}=d^{2}+a$, where

$$
a=|x|+\left|x^{\prime}\right|-2 \sqrt{|x|\left|x^{\prime}\right|}+2\left(t^{\prime}-t\right)\left(\sqrt{\left|x^{\prime}\right|}-\sqrt{|x|}\right) .
$$

Since $|x| \leq\left|x^{\prime}\right|$ and

$$
\left|\sqrt{\left|x^{\prime}\right|}-\sqrt{|x|}\right|=\frac{|| x^{\prime}|-| x||}{\sqrt{\left|x^{\prime}\right|}+\sqrt{|x|}} \leq \frac{h}{\sqrt{\left|x^{\prime}\right|}}
$$


we get

$$
a \leq\left|x^{\prime}\right|-|x|+2\left(\left|t^{\prime}\right|+|t|\right) h / \sqrt{\left|x^{\prime}\right|} \leq h+8 r h \sqrt{M}=2 h .
$$

Furthermore,

$$
a \geq-2\left(\left|t^{\prime}\right|+|t|\right)\left(\sqrt{\left|x^{\prime}\right|}-\sqrt{|x|}\right) \geq-h,
$$

and hence $|a| \leq 2 h$. Since $D \geq h$ and $d \geq h$, this implies that

$$
|D-d|=\frac{\left|D^{2}-d^{2}\right|}{D+d} \leq \frac{|a|}{2 h} \leq 1
$$

3.5. Notation. For $r \geq 0$ we set

$$
A_{r}=\left\{(x, t) \in \mathrm{R}^{n}:|t| \leq r|x|\right\} .
$$

Thus $A_{r}=\mathrm{R}^{n} \backslash D\left(e_{n}, \alpha\right)$, where $D\left(e_{n}, \alpha\right)$ is the double cone defined in 2.7 and $\cot \alpha=r$. Setting $q=r / \sqrt{1+r^{2}}$ we can also write

$$
A_{r}=\left\{z \in \mathrm{R}^{n}:\left|z \cdot e_{n}\right| \leq q|z|\right\} .
$$

Observe that $r=q / \sqrt{1-q^{2}}$.

3.7. Lemma. Suppose that $M>0$ and that $x, x^{\prime} \in \mathrm{R}^{n}$ with $|x|<M,\left|x^{\prime}\right|>M$. Let $x^{\prime \prime}$ be the point of the line segment $\left[x, x^{\prime}\right]$ with $\left|x^{\prime \prime}\right|=M$. Then

$$
\left|x^{\prime}\right|\left|x-x^{\prime \prime}\right| \leq 2 M\left|x-x^{\prime}\right| .
$$

Proof. Set $s=\left|x^{\prime}\right|\left|x-x^{\prime \prime}\right| /\left|x-x^{\prime}\right|$. We consider three cases.

Case 1. $\left|x^{\prime}\right| \leq 2 M$. Since $\left|x-x^{\prime \prime}\right| \leq\left|x-x^{\prime}\right|$, we have $s \leq\left|x^{\prime}\right| \leq 2 M$.

Case 2. $\left|x^{\prime}\right| \leq\left|x-x^{\prime}\right|$. Now $s \leq\left|x-x^{\prime \prime}\right| \leq|x|+\left|x^{\prime \prime}\right| \leq 2 M$.

Case 3. $\left|x^{\prime}\right| \geq 2 M$ and $\left|x-x^{\prime}\right| \leq\left|x^{\prime}\right|$. Since $\left|x^{\prime \prime}-x^{\prime}\right| \geq\left|x^{\prime}\right|-M$, we have $\left|x-x^{\prime \prime}\right|=\left|x-x^{\prime}\right|-\left|x^{\prime \prime}-x^{\prime}\right| \leq M$. Thus

$$
s \leq \frac{M\left|x^{\prime}\right|}{\left|x^{\prime}\right|-|x|}=\frac{M}{1-|x| /\left|x^{\prime}\right|} \leq 2 M
$$

3.8. Lemma. Suppose that $M \geq 1$ and that $r=1 / 8 \sqrt{M}$. Then the map $g \mid A_{r}$ is a 1-nearisometry.

Proof. Let $z=(x, t), z^{\prime}=\left(x^{\prime}, t^{\prime}\right) \in A_{r}$ with $|x| \leq\left|x^{\prime}\right|$, and let $d$ and $D$ be as in 3.3. We must show that $|D-d| \leq 1$. If $\left|x^{\prime}\right| \leq M$, this follows from 3.4. If $|x| \geq M$, then $|D-d|=0$. It remains to consider the case $|x| \leq M \leq\left|x^{\prime}\right|$.

There is a point $z^{\prime \prime}=\left(x^{\prime \prime}, t^{\prime \prime}\right)$ of the line segment $\left[z, z^{\prime}\right]$ with $\left|x^{\prime \prime}\right|=M$. Since $|t| \leq r|x| \leq r M$, we obtain by 3.7

$$
\left|t^{\prime \prime}\right|=\left|\frac{\left|x^{\prime}-x^{\prime \prime}\right|}{\left|x^{\prime}-x\right|} t+\frac{\left|x-x^{\prime \prime}\right|}{\left|x^{\prime}-x\right|} t^{\prime}\right| \leq M r+2 M r=3 r\left|x^{\prime \prime}\right| .
$$


Hence $\left|g z^{\prime \prime}-g z\right| \leq\left|z^{\prime \prime}-z\right|+1$ by 3.4. Consequently,

$$
D \leq\left|g z^{\prime}-g z^{\prime \prime}\right|+\left|g z^{\prime \prime}-g z\right| \leq\left|z^{\prime}-z^{\prime \prime}\right|+\left|z^{\prime \prime}-z\right|+1=d+1 .
$$

It remains to show that $D \geq d-1$. Computing as in 3.4 we get $D^{2}=d^{2}+a$ where

$$
a \geq 2\left(t^{\prime}-t\right)(\sqrt{M}-\sqrt{|x|}) \geq-2 r\left(\left|x^{\prime}\right|+|x|\right)(\sqrt{M}-\sqrt{|x|}) .
$$

Arguing as in 3.4 we see that it suffices to show that $a \geq-2 h=-2\left|x^{\prime}-x\right|$. We show that $a \geq-h$.

Case 1. $M \leq h$. Now $\left|x^{\prime}\right|+|x| \leq\left|x^{\prime}-x\right|+2|x| \leq h+2 M \leq 3 h$, and hence

$$
a \geq-2 r \cdot 3 h \sqrt{M}=-6 r h / 8 r \geq-h .
$$

Case 2. $M \geq h$. Now $\left|x^{\prime}\right|+|x| \leq h+2 M \leq 3 M$. Since $M-|x| \leq h$, we get

$$
a \geq-6 r M \frac{M-|x|}{\sqrt{M}+\sqrt{|x|}} \geq-6 r h \sqrt{M}=-6 h / 8 \geq-h .
$$

3.9. Notation We fix a number $\lambda, 0<\lambda \leq 1$; later we let $\lambda \rightarrow 0$. Let $r$ be as in 3.8 and set $A^{\prime}(r, \lambda)=A_{r} \cup \bar{B}(\lambda)$. Define $f: A^{\prime}(r, \lambda) \rightarrow \mathrm{R}^{n}$ by

$$
f x= \begin{cases}g x & \text { for } x \in A_{r}, \\ 0 & \text { for } x \in \bar{B}(\lambda) \backslash A_{r} .\end{cases}
$$

3.10. Lemma. The map $f: A^{\prime}(r, \lambda) \rightarrow \mathrm{R}^{n}$ is a $(1+\lambda)$-nearisometry.

Proof. Let $z, z^{\prime} \in A^{\prime}(r, \lambda)$ and set $d=\left|z^{\prime}-z\right|, D=\left|f z^{\prime}-f z\right|$. If $z, z^{\prime} \in A_{r}$, then $|D-d| \leq 1$ by 3.8. If $z, z^{\prime} \in \bar{B}(\lambda) \backslash A_{r}$, then $|D-d|=d \leq 2 \lambda \leq 1+\lambda$. Finally, let $z \in \bar{B}(\lambda) \backslash A_{r}, z^{\prime} \in A_{r}$. Since $f \mid A_{r}$ is a 1-nearisometry by 3.8 and since $f(0)=0$, we have ||$f z^{\prime}|-| z^{\prime}|| \leq 1$, and hence

$$
|D-d|=|| f z^{\prime}|-| z-z^{\prime}|| \leq 1+|z| \leq 1+\lambda .
$$

3.11. Lemma. Let $f: A^{\prime}(r, \lambda) \rightarrow \mathrm{R}^{n}$ be as in 3.9, let $A \subset A^{\prime}(r, \lambda)$ be unbounded, and let $T: \mathrm{R}^{n} \rightarrow \mathrm{R}^{n}$ be a linear isometry with $d(T, f \mid A)<\infty$. Then $T \mid \operatorname{cd} A=\mathrm{id}$.

Proof. Let $u \in \operatorname{cd} A$. Set $K=d(T, f \mid A)$ and choose a sequence $\left(z_{j}\right)$ in $A$ such that $\left|z_{j}\right| \rightarrow \infty$ and $u_{j}=p z_{j} \rightarrow u$. For large $j$ we have $f z_{j}=z_{j}+w$ with $w=\sqrt{M} e_{n}$. Then $\left|T z_{j}-z_{j}-w\right| \leq K$, and hence

$$
\left|T u_{j}-u_{j}-w /\right| z_{j}|| \leq K /\left|z_{j}\right| .
$$

As $j \rightarrow \infty$, this implies $T u=u$.

We turn to the proof of part (1) $\Rightarrow(2)$ of Theorem 2.3. 
3.12. Theorem. If $A \subset \mathrm{R}^{n}$ is an unbounded set with the $c$-IAP, then $\mu(A) \geq$ $1 / 17 c$.

Proof. The theorem is trivially true for $n=1$, since then $\mu(A)=1$ for each unbounded set $A \subset \mathrm{R}$. Assume that $n \geq 2$. Set $X=\operatorname{cd} A$ and $L=\operatorname{span} X$. We first show that $L=\mathrm{R}^{n}$. Assume that $L \neq \mathrm{R}^{n}$. We may assume that $L \subset \mathrm{R}^{n-1}$. Let $P: \mathrm{R}^{n} \rightarrow L$ and $P^{\prime}: \mathrm{R}^{n} \rightarrow L^{\perp}$ be the orthogonal projections. Set $M=25 c^{2}$ and $r=1 / 8 \sqrt{M}=1 / 40 c$. The set $Q=\left\{x \in A:\left|P^{\prime} x\right|>r|P x|\right\}$ is bounded by 3.1. Choose a number $R>0$ with $Q \subset \bar{B}(R)$. Since the property $c$-IAP is a similarity invariant by [Vä1, 3.1], we may replace $A$ by $A / R$ and thus assume that $Q \subset \bar{B}(1)$.

Choose $z \in A$ with $|P z| \geq M$. Write $P^{\prime} z=t e$ with $t \geq 0,|e|=1$. Using an auxiliary orthogonal map keeping $L$ fixed we may assume that $e=e_{n}$. Then $z=x+t e_{n}$ with $x=P z \in \mathrm{R}^{n-1}$.

Let $f: A^{\prime}(r, 1) \rightarrow \mathrm{R}^{n}$ be as in 3.9. By 3.10 this map is a 2-nearisometry. Since $A \subset A^{\prime}(r, 1)$ and since $A$ has the $c$-IAP, there is an isometry $T: \mathrm{R}^{n} \rightarrow \mathrm{R}^{n}$ with $d(T, f \mid A) \leq 2 c$. Since $f(0)=0$, there is a linear isometry $U: \mathbf{R}^{n} \rightarrow \mathbf{R}^{n}$ with $d(U, f \mid A) \leq 4 c$. By 3.11 we have $U \mid L=$ id. Since

$$
U z=U\left(x+t e_{n}\right)=x+t U e_{n}, \quad f z=x+(t+\sqrt{M}) e_{n},
$$

we obtain

$$
4 c \geq|f z-U z|=\left|(t+\sqrt{M}) e_{n}-t U e_{n}\right| \geq\left|(t+\sqrt{M}) e_{n}\right|-\left|t U e_{n}\right|=\sqrt{M}=5 c,
$$

a contradiction. We have proved that $\operatorname{span} X=\mathrm{R}^{n}$.

Set $q=1 / 17 c$ and $r=q / \sqrt{1-q^{2}}$. Since $c \geq 1 / 2$ (see 1.1), we have $r \leq 1 / 8$ and $M=1 / 64 r^{2} \geq 1$. Assume that $\mu(A)<q$. Choose $e \in S(1)$ such that $|u \cdot e| \leq q$ for all $u \in X$. We may assume that $e=e_{n}$. By 3.1 and 3.2.1, the set $A \backslash A_{r}$ is bounded; say $A \backslash A_{r} \subset \bar{B}(R)$. Arguing as above, we may replace $A$ by $\lambda A / R$; then $A \backslash A_{r} \subset \bar{B}(\lambda)$. Define the $(1+\lambda)$-nearisometry $f: A^{\prime}(r, \lambda) \rightarrow \mathrm{R}^{n}$ as in 3.9. As above, there is a linear isometry $U: \mathrm{R}^{n} \rightarrow \mathrm{R}^{n}$ with $d(U, f \mid A) \leq 2 c(1+\lambda)$. Since span $X=\mathrm{R}^{n}$, we have $U=$ id by 3.11 .

Choose a point $z=(x, t) \in A$ with $|x| \geq M$. By the definition 3.3 of $g$, we have $|f z-z|=|g z-z|=\sqrt{M}=1 / 8 r$. On the other hand, $|f z-z|=|f z-U z| \leq$ $2 c(1+\lambda)$. Hence $2 c(1+\lambda) \geq 1 / 8 r$. As $\lambda \rightarrow 0$, this yields $r \geq 1 / 16 c$. Since $r \leq 1 / 8$, this implies that $q=r / \sqrt{1+r^{2}} \geq 1 / 2 c \sqrt{65}>1 / 17 c$, a contradiction.

\section{Proof for $(2) \Rightarrow(1)$}

4.1. Outline of the proof. We consider the more general problem where $E$ and $F$ are arbitrary Hilbert spaces and $A$ is an unbounded subset of $E$ with $\mu(A) \geq$ $1 / c^{\prime}$. Suppose that $f: A \rightarrow F$ is an $\varepsilon$-nearisometry. We shall show that there is an isometry $T: E \rightarrow F$ such that $d(T, P f) \leq \sqrt{2} \varepsilon$ where $P$ is the orthogonal projection of $F$ onto $T E$. The part $(2) \Rightarrow(1)$ of 2.3 is then an immediate corollary. 
We normalize the situation by the conditions $0 \in A$ and $f(0)=0$. Set $X=\operatorname{cd} A$. We define a map $\varphi: X \rightarrow S(1)=S_{F}(1)$ as follows. Let $u \in X$. Choose a sequence $\left(x_{j}\right)$ in $A$ converging directionally to $u$. We show that the sequence $\left(p f x_{j}\right)$ converges to a point $u^{\prime} \in S(1)$ and that $u^{\prime}$ is independent of the choice of the sequence $\left(x_{j}\right)$. Setting $\varphi u=u^{\prime}$ we obtain the map $\varphi$. This map is an isometry, and it extends to a linear isometry $U: E \rightarrow F$. We show that $d(U, P f) \leq 2 c^{\prime} \varepsilon$, where $P: F \rightarrow U E$ is the projection. Combining $U$ and a translation we obtain the desired isometry $T$ with $T E=U E$ and $d(T, P f) \leq \sqrt{2} c^{\prime} \varepsilon$.

4.2. Notation. In this section $E$ and $F$ will always denote real Hilbert spaces of dimension at least one.

4.3. Lemma. Suppose that $\varepsilon>0$ and that $x, y \in E \backslash B(2 \varepsilon)$. Suppose also that $f:\{0, x, y\} \rightarrow F$ is an $\varepsilon$-nearisometry with $f(0)=0$. Then

$$
|p f x-p f y|^{2} \leq 4|p x-p y|^{2}+24 \varepsilon /|x|+24 \varepsilon /|y|+12 \varepsilon^{2} /|x||y| .
$$

Proof. Set

$$
s=|x|, t=|y|, r=|x-y|, \quad s^{\prime}=|f x|, t^{\prime}=|f y|, r^{\prime}=|f x-f y| .
$$

Since

$$
|a||b||p a-p b|^{2}=|a-b|^{2}-(|a|-|b|)^{2}
$$

for all $a, b \in E \backslash\{0\}$, and since $|t-s| \leq r,\left|t^{\prime}-s^{\prime}\right| \leq r^{\prime}$, we obtain

$$
\begin{aligned}
& s^{\prime} t^{\prime}|p f x-p f y|^{2}-s t|p x-p y|^{2} \\
& =\left(r+r^{\prime}\right)\left(r^{\prime}-r\right)+\left(t+t^{\prime}-s-s^{\prime}\right)\left(t-t^{\prime}+s^{\prime}-s\right) \\
& \leq\left(r+r^{\prime}\right)\left|r^{\prime}-r\right|+\left(|t-s|+\left|t^{\prime}-s^{\prime}\right|\right)\left(\left|t^{\prime}-t\right|+\left|s^{\prime}-s\right|\right) \\
& \leq\left(r+r^{\prime}\right)\left(\left|r^{\prime}-r\right|+\left|t^{\prime}-t\right|+\left|s^{\prime}-s\right|\right) \leq(2 r+\varepsilon) 3 \varepsilon .
\end{aligned}
$$

Since $r \leq s+t, s^{\prime} \geq s-\varepsilon \geq s / 2$ and $t^{\prime} \geq t-\varepsilon \geq t / 2$, this implies the lemma.

4.4. Lemma. Suppose that $A \subset E$ is unbounded, that $f: A \rightarrow F$ is a nearisometry with $f(0)=0$, and that $u \in \operatorname{cd} A$. Choose a sequence $\left(x_{j}\right)$ in $A$ converging directionally to $u$. Then the sequence $\left(p f x_{j}\right)$ converges to a point $u^{\prime} \in S(1)$, and $u^{\prime}$ is independent of the choice of the sequence $\left(x_{j}\right)$.

Proof. Since the sequence $\left(p x_{j}\right)$ is Cauchy and since $\left|x_{j}\right| \rightarrow \infty$, it follows from 4.3 that the sequence $\left(p f x_{j}\right)$ is Cauchy and hence convergent. If $\left(y_{j}\right)$ is another sequence in $A$ converging directionally to $u$, then so is the sequence $\left(z_{j}\right)=$ $\left(x_{1}, y_{1}, x_{2}, y_{2}, \ldots\right)$. Since the sequence $\left(p f z_{j}\right)$ is convergent, the subsequences $\left(p f x_{j}\right)$ and $\left(p f y_{j}\right)$ converge to the same limit.

We next prove an elementary but useful inequality. 
4.5. Lemma. Suppose that $x, y \in E$ and that $f:\{0, x, y\} \rightarrow F$ is an $\varepsilon$-nearisometry with $f(0)=0$. Then

$$
|f x \cdot f y-x \cdot y| \leq 2 \varepsilon(|x|+|y|+\varepsilon) .
$$

Proof. Since

$$
2 x \cdot y=|x|^{2}+|y|^{2}-|x-y|^{2}, \quad 2 f x \cdot f y=|f x|^{2}+|f y|^{2}-|f x-f y|^{2},
$$

we get

$$
\begin{aligned}
2|f x \cdot f y-x \cdot y| & \leq|| f x|-| x||(|f x|+|x|)+|| f y|-| y||(|f y|+|y|) \\
& +|| f x-f y|-| x-y||(|f x-f y|+|x-y|) \\
& \leq \varepsilon(2|x|+\varepsilon)+\varepsilon(2|y|+\varepsilon)+\varepsilon(2|x-y|+\varepsilon) \\
& \leq \varepsilon(4|x|+4|y|+3 \varepsilon),
\end{aligned}
$$

and the lemma follows.

4.6. Lemma. Suppose that $0 \in Y \subset E$ and that $\overline{\operatorname{span}} Y=E$. Suppose also that $\varphi: Y \rightarrow F$ is an isometry with $\varphi(0)=0$. Then there is a unique extension of $\varphi$ to an isometry $T: E \rightarrow F$. Moreover, $T$ is linear.

Proof. This result is well known. For the finite-dimensional case, see $R$, p. 9]. The general case follows easily from this by considering restrictions of $\varphi$ to finite subsets of $Y$ and making use of the uniqueness of $T$.

4.7. Theorem. Suppose that $A \subset E$ is an unbounded set such that $0 \in A$ and $\mu(A) \geq 1 / c^{\prime}$. Suppose also that $f: A \rightarrow F$ is an $\varepsilon$-nearisometry with $f(0)=0$. Then there is a linear isometry $U: E \rightarrow F$ with $d(U, P f) \leq 2 c^{\prime} \varepsilon$, where $P: F \rightarrow$ $U E$ is the orthogonal projection.

Proof. Set $X=\operatorname{cd} A$ and let $u \in X$. Let $\left(x_{j}\right)$ be a sequence in $A$ converging directionally to $u$. By 4.4, the sequence $\left(p f x_{j}\right)$ converges to a point $u^{\prime} \in S(1)$, and $u^{\prime}$ is independent of the choice of $\left(x_{j}\right)$. Setting $\varphi u=u^{\prime}$ we thus obtain a well defined map $\varphi: X \rightarrow S(1)$. We show that $\varphi$ is an isometry.

Since $f(0)=0$ and since $f$ is an $\varepsilon$-nearisometry, we have ||$f x|/| x|-1| \leq \varepsilon /|x|$ for all $x \in A \backslash\{0\}$. Hence $\left|f x_{j}\right| /\left|x_{j}\right| \rightarrow 1$ whenever $x_{j} \in A$ and $\left|x_{j}\right| \rightarrow \infty$. Consequently,

$$
\varphi u=\lim _{j \rightarrow \infty} \frac{f x_{j}}{\left|x_{j}\right|}
$$

for each sequence $\left(x_{j}\right)$ in $A$ converging directionally to $u$.

Let $u, v \in X$ and choose sequences $\left(x_{j}\right),\left(y_{j}\right)$ in $A$ converging directionally to $u$ and $v$, respectively. By 1.5 we have

$$
\left|f x_{j} \cdot f y_{j}-x_{j} \cdot y_{j}\right| \leq 2 \varepsilon\left(\left|x_{j}\right|+\left|y_{j}\right|+\varepsilon\right) .
$$


Dividing by $\left|x_{j}\right|\left|y_{j}\right|$ and letting $j \rightarrow \infty$ yields $\varphi u \cdot \varphi v=u \cdot v$. Since the vectors $u, v, \varphi u, \varphi v$ have norm one, this implies that $|\varphi u-\varphi v|=|u-v|$, and thus $\varphi$ is an isometry.

Extend $\varphi$ to an isometry $\varphi_{0}: X \cup\{0\} \rightarrow$ by $\varphi_{0}(0)=0$. Since $\mu_{1}(X)=$ $\mu(A)>0$, we have $\overline{\operatorname{span}} X=E$. By 4.6, the map $\varphi_{0}$ extends to a linear isometry $U: E \rightarrow F$. It remains to show that $d(U, P f) \leq 2 c^{\prime} \varepsilon$.

Let $U_{1}: E \rightarrow U E$ be the bijective linear isometry defined by $U$. Replacing $f$ by $f U_{1}^{-1}: U E \rightarrow F$ we may assume that $E \subset F$ and $U=$ id. Let $x \in E$ and set $\alpha=|P f x-x|$. We must show that $\alpha \leq 2 c^{\prime} \varepsilon$.

Choose a unit vector $e$ with $P f x-x=\alpha e$ and let $\lambda>1$. By the definition 2.2 of $\mu(A)$, there is $u \in X$ with $|u \cdot e| \geq \mu(A) / \lambda \geq 1 / \lambda c^{\prime}$. Choose a sequence $\left(x_{j}\right)$ in $A$ converging directionally to $u$. Then $f x_{j} /\left|x_{j}\right| \rightarrow U u=u$. By 4.5 we have

$$
\left|f x \cdot f x_{j}-x \cdot x_{j}\right| \leq 2 \varepsilon\left(|x|+\left|x_{j}\right|+\varepsilon\right) .
$$

Dividing by $\left|x_{j}\right|$ and letting $j \rightarrow \infty$ yields $|f x \cdot u-x \cdot u| \leq 2 \varepsilon$. Since $P f x \cdot u=f x \cdot u$, this implies that

$$
\alpha / \lambda c^{\prime} \leq \alpha|e \cdot u|=|(P f x-x) \cdot u| \leq 2 \varepsilon
$$

As $\lambda \rightarrow 1$, this gives $\alpha \leq 2 c^{\prime} \varepsilon$.

4.9. Corollary. Let $f: A \rightarrow F$ be as in 4.7. Then there is a linear map $S: F \rightarrow$ $E$ such that $|S|=1$ and $d(S f$, id $) \leq 2 c^{\prime} \varepsilon$.

Proof. Set $S=U_{1}^{-1} P$.

4.10. Remark. In the case where $A=E$ and thus $\mu(A)=1$, Corollary 4.9 was proved by [Qi, Th. 8] with the bound $6 \varepsilon$. A direct proof for this case with the bound $2 \varepsilon$ is given in Section 5; see 5.4 .

4.11. Corollary. Suppose that $A \subset \mathrm{R}^{n}$ is an unbounded set such that $0 \in A$ and $\mu(A) \geq 1 / c^{\prime}$. Suppose also that $f: A \rightarrow \mathrm{R}^{n}$ is an $\varepsilon$-nearisometry with $f(0)=0$. Then there is a linear isometry $U: \mathrm{R}^{n} \rightarrow \mathrm{R}^{n}$ with $d(U, f) \leq 2 c^{\prime} \varepsilon$.

4.12. The Jung constant. The Jung constant $J(V)$ of a normed space $V$ is the infimum of all $r>0$ such that every set $Q \subset V$ with $d(Q) \leq 2$ is contained in a ball of radius $r$. We have always $1 \leq J(V) \leq 2$, and $J\left(\mathrm{R}^{n}\right)=\sqrt{2 n /(n+1)}$ by the classical result of H.W.E. Jung [Ju]. Furthermore, $J(E)=\sqrt{2}$ for infinitedimensional Hilbert spaces $E$; see [Da, Th. 2] or [SE, p. 704].

4.13. Theorem. Suppose that $A \subset E$ is an unbounded set with $\mu(A) \geq 1 / c^{\prime}>$ 0 . Let $f: A \rightarrow F$ be an $\varepsilon$-nearisometry. Then there is an isometry $T: E \rightarrow F$ onto a closed linear subspace of $F$ such that

$$
d(T, P f) \leq J(F) c^{\prime} \varepsilon \leq \sqrt{2} c^{\prime} \varepsilon,
$$

where $P: F \rightarrow T E$ is the orthogonal projection. 
Proof. We follow the idea of [S], 1.2]. For each $a \in A$ we define $g_{a}: A-a \rightarrow F$ by $g_{a} x=f(x+a)-f a$. Then $0 \in A-a$ and $g_{a}(0)=0$. Let $U_{a}: E \rightarrow F$ be the linear isometry given by 4.7 for the map $g_{a}$.

Let $u \in \operatorname{cd} A$, and let $\left(x_{j}\right)$ be a sequence in $A$ converging directionally to $u$. Then $u \in \operatorname{cd}(A-a)$ and $\left(x_{j}-a\right)$ converges directionally to $u$. By (4.8) we have

$$
U_{a} u=\lim _{j \rightarrow \infty} \frac{g_{a}\left(x_{j}-a\right)}{\left|x_{j}-a\right|}=\lim _{j \rightarrow \infty} \frac{f x_{j}-f a}{\left|x_{j}-a\right|}=\lim _{j \rightarrow \infty} \frac{f x_{j}}{\left|x_{j}\right|},
$$

and hence $U_{a} u$ is independent of $a$ for each $u \in \operatorname{cd} A$. Since $\overline{\operatorname{span}} \operatorname{cd} A=E$, it follows that $U_{a}$ is independent of $a$, and we write $U=U_{a}$.

Let $P: F \rightarrow U E$ be the orthogonal projection and set $h=f-U: A \rightarrow F$. Then $d\left(U, P g_{a}\right) \leq 2 c^{\prime} \varepsilon$ for each $a \in A$. For all $a, b \in A$ we have

$$
|P h a-P h b|=\left|P g_{b}(a-b)-U_{b}(a-b)\right| \leq 2 c^{\prime} \varepsilon .
$$

Hence $d(P h A) \leq 2 c^{\prime} \varepsilon$. Let $\lambda>1$. Then $P h A$ is contained in the ball $\bar{B}\left(w, \lambda J(F) c^{\prime} \varepsilon\right)$ for some $w \in U E$. Setting $T x=U x+w$ we obtain an isometry $T: E \rightarrow F$ with $T E=U E$. For each $x \in A$ we have

$$
|T x-\operatorname{Pf} x|=|w-P h x| \leq \lambda J(F) c^{\prime} \varepsilon .
$$

As $\lambda \rightarrow 1$, this gives the theorem.

4.14. Corollary. Part (2) $\Rightarrow$ (1) of Theorem 2.3 is true.

\section{A short proof for the Hyers-Ulam theorem}

The proof of the original Hyers-Ulam theorem in $\mathrm{HU}$ is elementary but rather long. We next give a considerably shorter elementary proof, which leads to the optimal constant and also gives the finite-dimensional version due to BhatiaŠemrl BS, Th. 1]. A crucial tool is our inequality 4.5. Otherwise, the proof is self-contained.

In 5.5 we give an improved version of the Hyers-Ulam theorem with relaxed surjectivity condition.

5.1. Theorem. Suppose that $E$ is a Banach space, that $F$ is a Hilbert space, and that $f: E \rightarrow F$ is an $\varepsilon$-nearisometry with $f(0)=0$. Then there is a linear isometry $T: E \rightarrow F$ such that $d(T, P f) \leq 2 \varepsilon$, where $P: F \rightarrow T E$ is the orthogonal projection. Hence $E$ is isomorphic to a Hilbert space. $2 \varepsilon$.

If $f$ is surjective or if $\operatorname{dim} E=\operatorname{dim} F<\infty$, then $T$ is surjective and $d(T, f) \leq$

Proof. We first show that the limit $T x=\lim _{s \rightarrow \infty} f(s x) / s$ exists for each $x \in E$. Since $F$ is complete, it suffices to show that

$$
\sup \{|f(s x) / s-f(t x) / t|: t \geq s\} \rightarrow 0
$$


as $s \rightarrow \infty$. Let $0<s \leq t$. By 4.5 we obtain

$$
\begin{aligned}
& |f(s x) / s-f(t x) / t|^{2}=|f(s x)|^{2} / s^{2}+|f(t x)|^{2} / t^{2}-2 f(s x) \cdot f(t x) / s t \\
& \leq(s|x|+\varepsilon)^{2} / s^{2}+(t|x|+\varepsilon)^{2} / t^{2}-2|x|^{2}+4 \varepsilon(|x| / t+|x| / s+\varepsilon / s t) \\
& \leq 12 \varepsilon|x| / s+6 \varepsilon^{2} / s^{2}
\end{aligned}
$$

which implies (5.2). Observe that $T(0)=0$.

We next show that the map $T: E \rightarrow F$ is an isometry and hence linear. Let $x, y \in E$. Since $f$ is an $\varepsilon$-nearisometry, we have

$$
|| f(s x)-f(s y)|-| s x-s y|| \leq \varepsilon .
$$

Dividing by $s$ and letting $s \rightarrow \infty$ yields $|T x-T y|=|x-y|$.

Let $T_{1}: E \rightarrow T E$ be the bijective linear isometry defined by $T$. Replacing $f$ by $f T_{1}^{-1}: T E \rightarrow F$ we may assume that $E \subset F$ and that $T=$ id. Let $P: F \rightarrow E$ be the orthogonal projection and let $x \in E$. We must show that

$$
|P f x-x| \leq 2 \varepsilon
$$

Set $\alpha=|P f x-x|$ and choose a unit vector $u \in E$ with $\alpha u=P f x-x$. By 4.5 we have

$$
|f x \cdot f(s u)-x \cdot s u| \leq 2 \varepsilon(|x|+s+\varepsilon)
$$

for all $s>0$. Since $f(s u) / s \rightarrow T u=u$ as $s \rightarrow \infty$, this yields $|f x \cdot u-x \cdot u| \leq 2 \varepsilon$. Since $f x \cdot u=P f x \cdot u$, we obtain

$$
\alpha=\alpha u \cdot u=f x \cdot u-x \cdot u \leq 2 \varepsilon,
$$

and (5.3) follows.

If $\operatorname{dim} E=\operatorname{dim} F<\infty$, then $T$ is surjective. Assume that $f$ is surjective and that $E \neq F$. Choose a unit vector $e \in E^{\perp}$ and then $x \in E$ with $f x=$ $(3 \varepsilon+1) e$. Since $f$ is an $\varepsilon$-nearisometry and since $P f x=0$, we obtain by (5.3) the contradiction

$$
3 \varepsilon+1=|f x| \leq|x|+\varepsilon \leq 3 \varepsilon
$$

5.4. Remark. Setting $S=T_{1}^{-1} P$ in 5.1 we obtain a linear map $S: F \rightarrow E$ with $|S|=1$ and $d(S f$, id $) \leq 2 \varepsilon$.

We finally show that the surjectivity condition of 5.1 can be replaced by the weaker condition $\tau(f E)<1$, where $\tau$ was defined in 2.7. Observe that $\tau(F)=0$.

5.5. Theorem. Suppose that $E$ is a Banach space, that $F$ is a Hilbert space, and that $f: E \rightarrow F$ is an $\varepsilon$-nearisometry such that $f(0)=0$ and $\tau(f E)<1$. Then there is a surjective linear isometry $T: E \rightarrow F$ with $d(T, f) \leq 2 \varepsilon$. 
Proof. The proof of 5.1 is valid until the last paragraph. Assume again that $E \neq F$, and choose a unit vector $e \in E^{\perp}$. Choose a number $q$ with $\tau(f E)<q<$

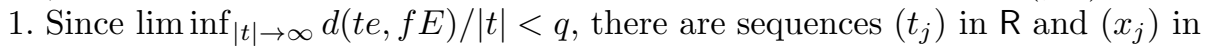
$E$ such that $\left|t_{j}\right| \rightarrow \infty$ and

$$
\left|t_{j} e-f x_{j}\right| \leq q\left|t_{j}\right|
$$

for all $j$. Setting $y_{j}=f x_{j}$ we have

$$
(1-q)\left|t_{j}\right| \leq\left|y_{j}\right| \leq(1+q)\left|t_{j}\right| \leq 2\left|t_{j}\right|
$$

for all $j$.

Since $\left|P y_{j}-x_{j}\right| \leq 2 \varepsilon$ by (5.3), we have $\left|P y_{j}\right| \geq\left|x_{j}\right|-2 \varepsilon \geq\left|y_{j}\right|-3 \varepsilon$. Since $\left|y_{j}\right| \rightarrow \infty$ by (5.7), we have $\left|y_{j}\right| \geq 3 \varepsilon$ for large $j$, and then $\left|P y_{j}\right|^{2} \geq\left|y_{j}\right|^{2}-6 \varepsilon\left|y_{j}\right|$. Since $\left|y_{j}\right|^{2}=\left|P y_{j}\right|^{2}+\left|y_{j}-P y_{j}\right|^{2}$, we get

$$
e \cdot y_{j}=e \cdot\left(y_{j}-P y_{j}\right) \leq\left|y_{j}-P y_{j}\right| \leq \sqrt{6 \varepsilon\left|y_{j}\right|} \leq 4 \sqrt{\varepsilon t_{j}}
$$

by (5.7). Since (5.6) implies that

$$
t_{j}^{2}+\left|y_{j}\right|^{2}-2 t_{j} e \cdot y_{j} \leq q^{2} t_{j}^{2}
$$

we obtain $\left(1-q^{2}\right) t_{j}^{2} \leq 8 t_{j} \sqrt{\varepsilon t_{j}}$. Dividing by $t_{j}^{2}$ and letting $j \rightarrow \infty$ gives the desired contradiction.

5.8. Corollary. If $f: E \rightarrow F$ is a nearisometry, then $\tau(f E) \in\{0,1\}$.

Proof. Since $\tau$ is invariant under translations, we may assume that $f(0)=0$. If $\tau(f E)<1$, it follows from 5.5 that $d(y, f E) \leq 2 \varepsilon$ for all $y \in F$, and hence $\tau(f E)=0$.

\section{A remark on Banach spaces}

The proofs in the preceding sections make substantial use of the inner product, and there seems to be no easy way to extend them for general Banach spaces. However, we show that instead of considering surjective nearisometries between Banach spaces $E, F$, it suffices to consider maps $f: A \rightarrow F$ such that the sets $E \backslash A$ and $F \backslash f A$ are bounded. This means that the theorem of Hyers-UlamGevirtz-Omladič-Šemrl [BD, 15.2] for Banach spaces is not, after all, a global result but a local property of maps near the point $\infty$. The result was suggested to the author by O. Martio.

6.1. Theorem. Suppose that $E$ and $F$ are Banach spaces, that $A \subset E$, and that $f: A \rightarrow F$ is an $\varepsilon$-nearisometry such that the sets $E \backslash A$ and $F \backslash f A$ are bounded. Then there is a surjective isometry $T: E \rightarrow F$ with $d(T, f) \leq 2 \varepsilon$. For each $x_{0} \in A$ we can choose $T$ so that $T x_{0}=f x_{0}$. 
Proof. We may assume that $x_{0}=0, f x_{0}=0$. Choose a number $R>\varepsilon$ such that $E \backslash A \subset B(R)$ and $F \backslash f A \subset B(2 R)$. Define $f_{1}: E \rightarrow F$ by $f_{1} x=2 x$ for $|x| \leq R$ and by $f_{1} x=f x$ for $|x|>R$. Then $f_{1}$ is a surjective $K$-nearisometry with $K=3 R+\varepsilon$. By [BD, 15.2], there is a linear surjective isometry $T: E \rightarrow F$ with $d\left(T, f_{1}\right) \leq 2 K$. Hence $d(T, f)<\infty$. Now an easy modification of the proof of [BU, 15.2] (see [BD, p. 362]) shows that $d(T, f) \leq 2 \varepsilon$.

If $E$ and $F$ are Banach spaces of the same finite dimension, then every $\varepsilon$ nearisometry $f: E \rightarrow F$ with $f(0)=0$ can be approximated by a linear isometry $T: E \rightarrow F$ with $d(T, f) \leq 2 \varepsilon$ [Di, Th. 1].

6.2. Open problem. Suppose that $E$ and $F$ are finite-dimensional Banach spaces with $\operatorname{dim} E=\operatorname{dim} F<\infty$, that $A \subset E$ is a half space and that $f: A \rightarrow F$ is an $\varepsilon$-nearisometry. Does there exist an isometry $T: E \rightarrow F$ such that $d(T, f) \leq K \varepsilon$ with some universal constant $K$ ?

\section{References}

[ATV] P. Alestalo, D.A. Trotsenko and J. Väisälä, Isometric approximation, Israel J. Math. 125 (2001), 61-82.

[BL] Y. Benyamini and J. Lindenstrauss, Geometric nonlinear functional analysis I, AMS Colloquium Publications 48, 2000.

[BŠ] R. Bhatia and P. Šemrl, Approximate isometries on Euclidean spaces, Amer. Math. Monthly 104 (1997), 497-504.

[Da] J. Daneš, On the radius of a set in a Hilbert space, Comment. Math. Univ. Carolin. 25 (1984), 355-362.

[Di] S.J. Dilworth, Approximate isometries in finite-dimensional normed spaces, Bull. London Math. Soc. 31 (1999), 471-476.

[HV] T. Huuskonen and J. Väisälä, Hyers-Ulam constants of Hilbert spaces, preprint.

[HU] D.H. Hyers and S.M. Ulam, On approximate isometries, Bull. Amer. Math. Soc. 51 (1945), 288-292.

[Jo] F. John, Rotation and strain, Comm. Pure Appl. Math. 14 (1961), 391-413.

[Ju] H.W.E. Jung, Über die kleinste Kugel, die eine räumliche Figur einschliesst, J. Reine Angew. Math. 123 (1901), 241-257.

[Ma] E. Matoušková, Almost isometries of balls, preprint.

[Qi] S. Qian, $\varepsilon$-isometric embeddings, Proc. Amer. Math. Soc. 123 (1995), 1797-1803.

[Re] E.G. Rees, Notes on geometry, Springer, 1983.

[Še] P. Šemrl, Hyers-Ulam stability of isometries, Houston J. Math. 24 (1998), 699706.

[Vä1] J. Väisälä, Isometric approximation property in euclidean spaces, Israel J. Math., to appear.

[Vä2] J. Väisälä, A survey of nearisometries, Report. Univ. Jyväskylä, to appear. 
Preprints of the author can be downloaded from www.helsinki.fi $/{ }^{\sim}$ jvaisala/preprints.html.

Matematiikan laitos

Helsingin yliopisto

PL 4, Yliopistonkatu 5

00014 Helsinki, Finland

jvaisala@cc.helsinki.fi 